\title{
Pinhole X-ray fluorescence imaging of gadolinium and gold nanoparticles using polychromatic X-rays: a Monte Carlo study
}

This article was published in the following Dove Press journal:

International Journal of Nanomedicine

II August 2017

Number of times this article has been viewed

\author{
Seongmoon Jung ${ }^{1,2}$ \\ Wonmo Sung ${ }^{1,2}$ \\ Sung-Joon $\mathrm{Ye}^{1-3}$ \\ 'Department of Transdisciplinary \\ Studies, Graduate School of \\ Convergence Science and Technology, \\ Seoul National University, Seoul, \\ Republic of Korea; ${ }^{2}$ Biomedical \\ Research Institute, College of \\ Medicine, Seoul National University, \\ Seoul, Republic of Korea; ${ }^{3}$ Advanced \\ Institutes of Convergence Technology, \\ Seoul National University, Suwon, \\ Republic of Korea
}

\begin{abstract}
This work aims to develop a Monte Carlo (MC) model for pinhole K-shell X-ray fluorescence (XRF) imaging of metal nanoparticles using polychromatic X-rays. The MC model consisted of two-dimensional (2D) position-sensitive detectors and fan-beam X-rays used to stimulate the emission of XRF photons from gadolinium $(\mathrm{Gd})$ or gold $(\mathrm{Au})$ nanoparticles. Four cylindrical columns containing different concentrations of nanoparticles ranging from $0.01 \%$ to $0.09 \%$ by weight ( $\mathrm{wt} \%$ ) were placed in a $5 \mathrm{~cm}$ diameter cylindrical water phantom. The images of the columns had detectable contrast-to-noise ratios (CNRs) of 5.7 and 4.3 for $0.01 \mathrm{wt} \% \mathrm{Gd}$ and for $0.03 \mathrm{wt} \% \mathrm{Au}$, respectively. Higher concentrations of nanoparticles yielded higher CNR. For $1 \times 10^{11}$ incident particles, the radiation dose to the phantom was $19.9 \mathrm{mGy}$ for $110 \mathrm{kVp}$ X-rays (Gd imaging) and $26.1 \mathrm{mGy}$ for $140 \mathrm{kVp}$ X-rays (Au imaging). The MC model of a pinhole XRF can acquire direct 2D slice images of the object without image reconstruction. The MC model demonstrated that the pinhole XRF imaging system could be a potential bioimaging modality for nanomedicine.
\end{abstract}

Keywords: X-ray fluorescence, pinhole, nanoparticles, bioimaging, CNR, radiation dose

\section{Introduction}

The development and application of metal nanoparticles (MNPs) have become emerging research areas in medicine, known as nanomedicine. In particular, the applications of MNPs in cancer therapy and diagnosis include for tumor targeting, for drug and gene delivery, as imaging contrast agents, and as radiosensitizers. ${ }^{1-5}$ Among the various MNPs, gadolinium oxide nanoparticles (GdNPs) and gold nanoparticles (AuNPs) were proven to have the greatest potential for these purposes. For example, GdNPs can provide contrast enhancement for magnetic resonance imaging (MRI). ${ }^{6-8}$ In addition, there have been efforts to use GdNPs as therapeutic agents for radiation therapy. ${ }^{9,10}$ More recently, the radiosensitizing properties of GdNPs compared to those of Gd(III) chelates have been studied. ${ }^{11}$ Among all the MNPs, AuNPs were proven to be a much safer and less toxic sensitizing agent for cancer treatment. ${ }^{12}$ Since Hainfeld et $\mathrm{al}^{13}$ first experimentally demonstrated the feasibility of using AuNP as a radiosensitizer in 2004, numerous studies on AuNP-enhanced radiation therapy using external $\mathrm{X}$-ray sources, brachytherapy sources, as well as proton and carbon ion beams have been conducted. ${ }^{14-21}$ The mechanisms of the radiation sensitization effect and methodologies for characterizing such effects have also been investigated. ${ }^{22-24}$ The low-energy electrons emitted from AuNPs locally deposit their energies in close proximity, which results in physical radiation dose enhancement effect. ${ }^{22-25}$ In addition, the effects of 
AuNPs on the biological system (particularly on the cell cycle), metabolic activities, and DNA repair pathways have been reported. ${ }^{22,26-31}$ The dependency of radiation sensitization on the size, shape, and concentration of AuNPs has also been presented by several studies. ${ }^{23,27,32-36}$

In order to further develop the nanoparticle-enhanced radiation therapy, monitoring the biological effects and toxicity based on in vivo biodistributions and concentrations of the nanoparticles (NP) in the target and normal tissues must be carried out during preclinical studies. ${ }^{23}$ However, due to the lack of an effective in vivo imaging tool, there has been a slow progress on further studies in this area. ${ }^{37} \mathrm{X}$-ray fluorescence (XRF) computed tomography (XFCT) is one of the promising imaging modalities for in vivo imaging. ${ }^{38}$ XFCT was first proposed in 1986 using synchrotron sources. ${ }^{39}$ Since polychromatic X-ray sources were made available on the laboratory scale, implementing benchtop fluorescence imaging systems has shown that XFCT could be a potential molecular imaging device similar to positron emission tomography (PET) and single-photon emission computed tomography (SPECT). ${ }^{38}$ Focusing on K-shell XFCT, 0.5\% gold by weight (wt \%) was able to be detected using $105 \mathrm{kVp}$ cone-beam X-rays by Jones et al. ${ }^{40}$ Ahmad et al ${ }^{41}$ showed images of $0.25 \mathrm{wt} \%$ AuNP using $120 \mathrm{kVp}$ pencil-beam X-rays by optimizing the detector angular configuration. Recently, L-shell imaging of AuNP using a benchtop X-ray source has been developed to improve the detection limit on the order of parts per million (ppm). ${ }^{42-44}$ The previously mentioned studies used a single cadmium-telluride (CdTe) detector and a single silicon drift detector (SDD) for K-shell and L-shell imaging, respectively. However, the image acquisition time was long because it was necessary to rotate the phantoms and translate the detectors to obtain full three-dimensional (3D) images. The research by Meng et $\mathrm{al}^{45}$, Fu et $\mathrm{al}^{46}$, and Groll et $\mathrm{al}^{47}$ demonstrated improved images obtained by the pinhole-based system and 2D position-sensitive detectors computationally and experimentally. In these studies, pinhole collimation with a 2D X-ray charge-coupled device (CCD) camera (Model \#934N, Andor Technology), which has a detection efficiency of $\sim 30 \%$ at $10 \mathrm{keV}$ and $\sim 15 \%$ at $15 \mathrm{keV}$, was used to detect iron, zinc, and bromine solutions, whose energies of $\mathrm{K}$-shell fluorescence $\mathrm{X}$-rays range from $6.4 \mathrm{keV}$ to $13.3 \mathrm{keV}$. Very small samples (ie, $0.75 \mathrm{~mm}$ diameter cylindrical tubes) were used due to the limited penetration depth of these low-energy fluorescence X-rays. On the other hand, L-shell XFCT for AuNP can detect much lower concentrations than K-shell XFCT due to its much lower Compton background noise. However, the penetration depth of L-shell fluorescence limits the imaging technique to very small samples of $<1 \mathrm{~cm}$ in diameter or thickness. ${ }^{42-44}$ To perform XFCT for animal and human clinical studies, K-shell fluorescence images for MNPs would be necessary.

In this work, a Monte Carlo (MC) model was developed for pinhole K-shell XRF imaging of GdNPs and AuNPs using fan-beam polychromatic X-rays combined with a 2D array of position-sensitive detectors. XRF photons stimulated by incoming X-rays are isotropic sources, and thus, pinhole collimation is a good choice for imaging. Furthermore, the photons passing through a pinhole were expected to contain its position information, which enable the acquisition of a 2D slice image directly from one sheet of fan-beam irradiation without image reconstruction, translation, and rotation of the object. The MC model for this pinhole XRF system was quantitatively evaluated in terms of image quality and radiation dose for the polychromatic and monochromatic X-rays.

\section{Materials and methods MC model}

A pinhole XRF imaging system was modeled using MC N-Particle Version 6.1 (MCNP6.1), as illustrated in Figure $1 .{ }^{48}$ The MCNP code has been widely used for the simulation studies on cadmium-zinc-telluride (CZT), CdTe, and high-purity germanium (HPGe) semiconductor detectors for medical imaging. ${ }^{49-51}$ The pinhole XRF imaging system has an imaging mechanism very similar to SPECT. ${ }^{38}$ The model was benchmarked against the design and the dimension of the pinhole, as well as the type and the pixel size of the detector commonly used in preclinical pinhole SPECT. ${ }^{52-56}$ A cylindrical water phantom in the middle, as shown in Figure 1A, had a diameter of $5 \mathrm{~cm}$ and a height of $5 \mathrm{~cm}$ each. Four cylindrical water columns, $1 \mathrm{~cm}$ in diameter and $3 \mathrm{~cm}$ in height, containing GdNPs or AuNPs of different concentrations $(0.01 \mathrm{wt} \%, 0.03 \mathrm{wt} \%, 0.06 \mathrm{wt} \%$, and $0.09 \mathrm{wt} \%)$ were placed in the water phantom, as shown in Figure 1B. Two pinhole collimators with pinholes of $2 \mathrm{~mm}$ in diameter were located on both sides of the phantom on a lead sheet with a thickness of $5 \mathrm{~mm}$. The pinhole had a cone-shaped profile with an acceptance angle of $102^{\circ}$ (Figure S1). The beam plane-to-collimator and the collimator-to-detector distances were $2.5 \mathrm{~cm}$ each.

The incident X-ray beam was specified as a fan beam of $5 \mathrm{~mm}$ width and $5 \mathrm{~cm}$ length. Due to the high-energy resolution of the semiconductor detector, ${ }^{57}$ a commercial pixelated CZT gamma camera composed of four SRE4001-CZT25.4 modules (Integrated Detect Electronics AS, Oslo, Norway) was chosen as the detector model for the MC simulation. The detector consisted of $32 \times 32$ pixels, with each pixel having 
A

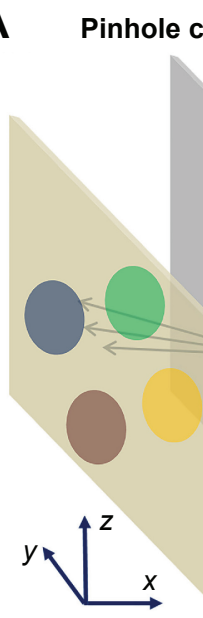

Pixelated detector
Pinhole collimator

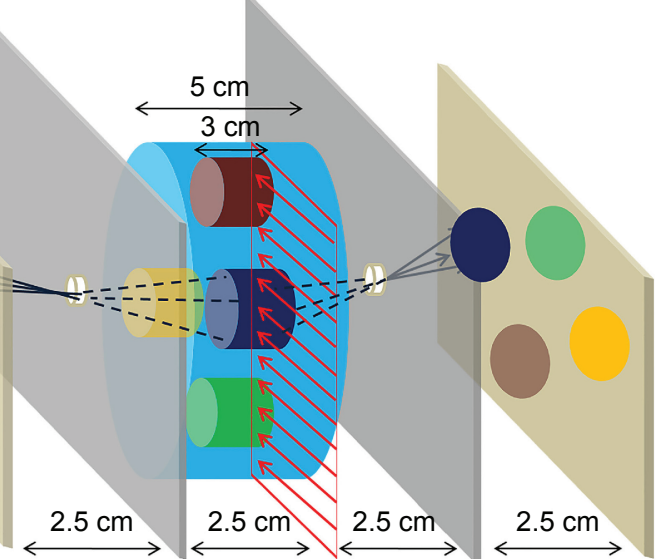

B

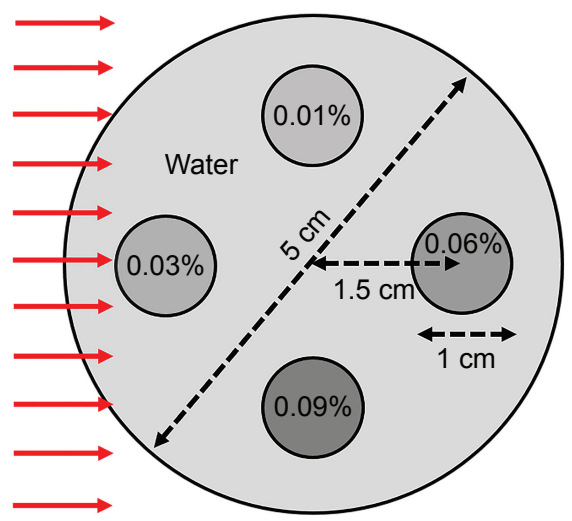

Figure I MC simulation geometry and imaging water phantom.

Notes: (A) MC simulation geometry for pinhole K-shell XRF imaging system and (B) imaging water phantom where four columns are assumed to have different concentrations of either gadolinium (Gd) or gold ( $\mathrm{Au}$ ) nanoparticles. The red arrows indicate the incident fan-beam X-rays. Gd or Au columns of 0.01 wt $\%, 0.03$ wt $\%, 0.06$ wt $\%$, and 0.09 wt $\%$ are located left, in, out, and right with respect to the incident direction of X-rays, respectively.

Abbreviations: MC, Monte Carlo; XRF, X-ray fluorescence; Gd, gadolinium; Au, gold; wt, weight.

a dimension of $1.6 \times 1.6 \mathrm{~mm}^{2}$ and a thickness of $5 \mathrm{~mm}$. The detector had an Al6061 window with a thickness of $1.5 \mathrm{~mm}$ in front of the CZT crystal. The geometric efficiency and system resolution were calculated as shown in the Supplementary materials. Utilizing the geometrical symmetry of the collimator-and-detector configuration (Figure 1A), MC images from both sides were combined to reduce the noise for a given computation time.

\section{X-ray sources}

To acquire Gd K-shell XRF images, $110 \mathrm{kVp}$ polychromatic $\mathrm{X}$-rays filtered with $0.3 \mathrm{~mm}$ tungsten (W) (denoted as 110 $\mathrm{kVp}$ ) were used. On the other hand, $140 \mathrm{kVp} X$-rays with $1.5 \mathrm{~mm}$ aluminum $(\mathrm{Al}), 0.25 \mathrm{~mm}$ copper $(\mathrm{Cu})$, and $0.75 \mathrm{~mm}$ tin (Sn) filtration (denoted as $140 \mathrm{kVp}$ ) were used for $\mathrm{Au}$
$\mathrm{K}$-shell XRF imaging. The filters used for $140 \mathrm{kVp} \mathrm{X-rays}$ were commercial filters of the X-RAD 320 (Precision X-ray Inc, North Branford, CT, USA). The energy spectra for the two polychromatic X-rays were extracted from the SpekCalc code that has been validated with measurements and $\mathrm{MC}$ results and were previously used in several studies. ${ }^{58-61}$ In addition, monochromatic X-rays of two different energies (ie, $51 \mathrm{keV}$ for $\mathrm{Gd}$ and $81 \mathrm{keV}$ for $\mathrm{Au}$ ) were used to compare the image quality and the radiation dose. The X-rays of $51 \mathrm{keV}$ and $81 \mathrm{keV}$ were expected to have more fluorescence emissions, since these energies are close to the $K_{\text {edge }}$ energies of Gd (ie, $50.2 \mathrm{keV}$ ) and $\mathrm{Au}$ (ie, $80.7 \mathrm{keV}$ ). The incident X-ray source spectra for $\mathrm{Gd}$ and $\mathrm{Au}$ are shown in Figure 2. Each simulation was conducted with $1 \times 10^{11}$ histories.
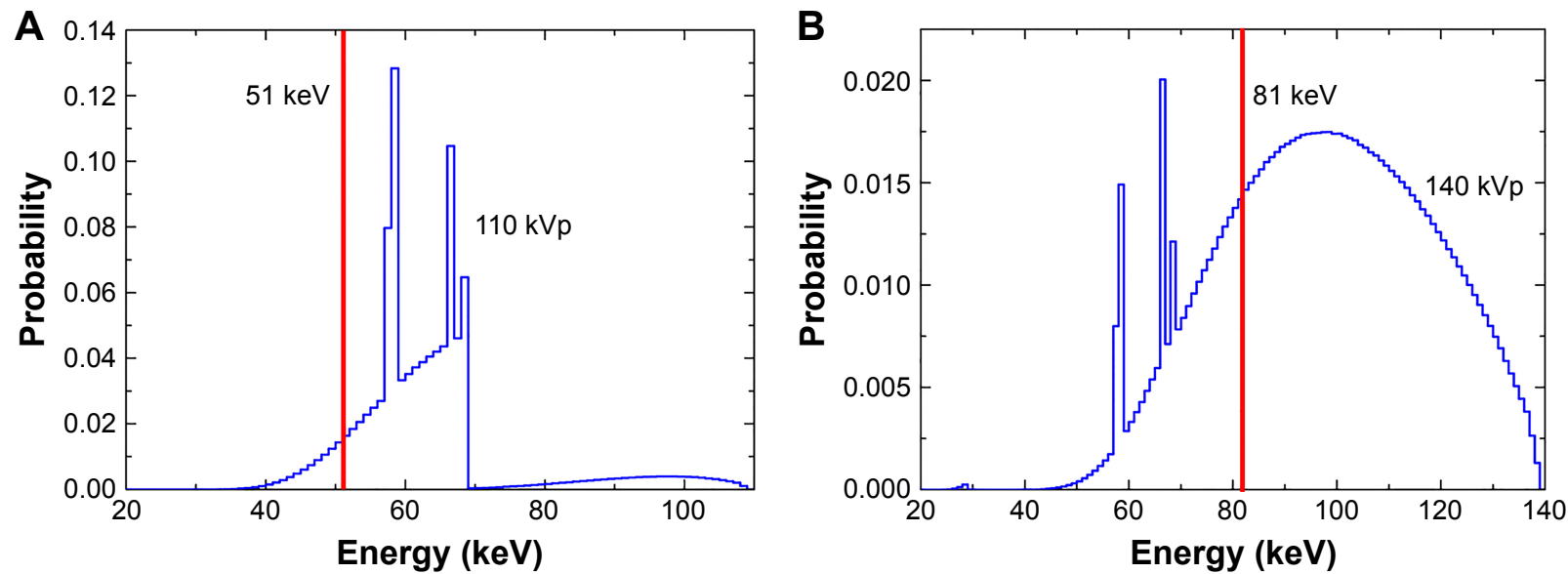

Figure 2 Incident X-ray source spectra for pinhole K-shell XRF imaging for (A) gadolinium and (B) gold. Abbreviation: XRF, X-ray fluorescence. 


\section{Image processing}

$\mathrm{XRF}$ and scattered photons reaching the detector array were tracked by the energy-specific pulse height tallies (ie, F8 of MCNP6.1). In order to detect the distinct K-shell XRF peaks above the Compton background counts, an energy bin of $1 \mathrm{keV}$ was set for the detector tallies. The $K_{\alpha 1}$ and $K_{\alpha 2}$ peaks for $\mathrm{Gd}$ and $\mathrm{Au}$ were acquired in the simulation. The peak energies of Gd were $42.4 \mathrm{keV}$ and $43.1 \mathrm{keV}$, while Au XRF lines resulted in peaks at $67.2 \mathrm{keV}$ and $69.0 \mathrm{keV} .{ }^{62}$

Since the energy resolution of CZT gamma camera was much higher than $1 \mathrm{keV}$, degradation of simulation results was needed to reflect realistic situations. We measured the energy spectrum of amerisium-241 (Am-241) radioisotope (Ortec, Oak Ridge, TN, USA) emitting $59.5 \mathrm{keV}$ gammas (Figure S2). Full width at half maximum (FWHM) at $59.5 \mathrm{keV}$ was assumed to not be significantly different from the \%FWHM at K-shell XRF energies of $\mathrm{Gd}(\sim 43 \mathrm{keV})$ and $\mathrm{Au}(\sim 68 \mathrm{keV})$. Degradation with the measured $\% \mathrm{FWHM}$ was applied for the MC-based $1 \mathrm{keV}$ spectra, which caused XRF peaks to be broadened (Figures S3 and S4). The raw fluorescence signals were derived by subtracting 41-46 keV (GdNP imaging) and 66-71 keV (AuNP imaging) photon counts of the pure water phantom from the photon counts at the same energies for the phantom inserted with Gd and Au columns.

The attenuation corrections for the incident beam and XRF photons were taken into account by using the mass attenuation coefficient published by the National Institute of Standards and Technology (NIST) ${ }^{63}$ which were derived using the following equation:

$$
N_{a}^{i}=N_{0}^{i}\left(1 / e^{-\mu_{1} y^{i}}\right)\left(1 / e^{-\mu_{K} x^{i}}\right)
$$

where $N_{a}^{i}$ is the attenuation corrected fluorescence photon count, $N_{0}^{i}$ is the raw fluorescence photon count of the $i$ th pixel, $\mu_{I}$ is the linear attenuation coefficient of water at the incident X-ray energy, and $\mu_{K}$ is the linear attenuation coefficient of water at K-shell XRF energy (ie, Gd: $43 \mathrm{keV}$ and $\mathrm{Au}: 68 \mathrm{keV}$ ). The midplane of the water phantom where the incident fan beam passed through was divided into $32 \times 32$ pixels similar to a pinhole projection of detector array. Since the beam plane-to-collimator and collimatorto-detector distances were the same, the photons from the $i$ th pixel of the midplane were assumed to be the photons arriving at the $i$ th pixel of the detector. $y^{i}$ is the path length of incident X-rays in the phantom, while $x^{i}$ indicates the path length of XRF photons of the $i$ th pixel in the phantom (Figure 3). For the polychromatic X-rays, the term $e^{-\mu_{I} y^{i}}$ was replaced with

$$
e^{-\mu_{I} y^{i}}=\left(\sum_{m=E_{K}}^{E_{\max }} N_{m} e^{-\mu_{I} y^{i}}\right) / \sum_{m=E_{K}}^{E_{\max }} N_{m}
$$

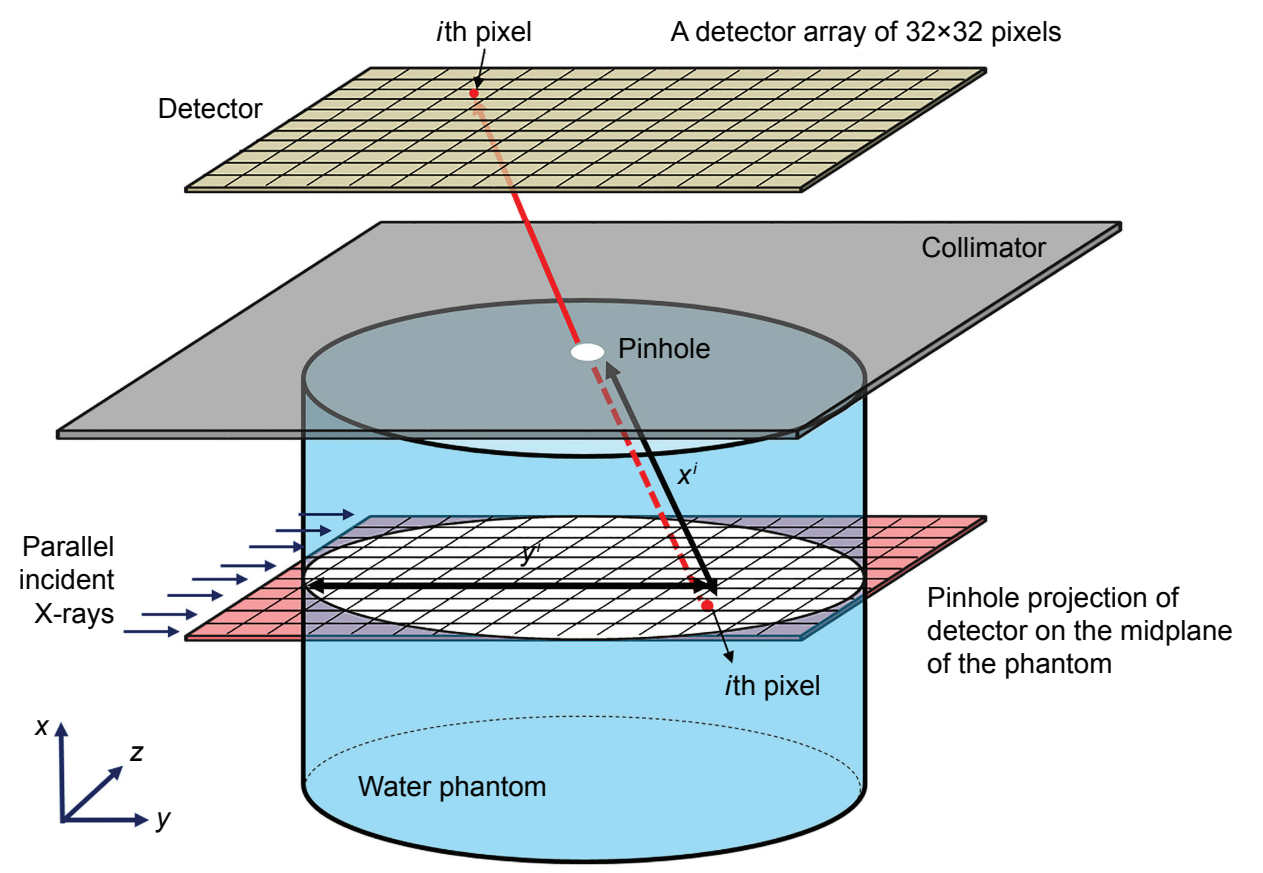

Figure 3 Schematic representation of pinhole XRF imaging system.

Notes: The midplane of the water phantom was divided into $32 \times 32$ pixels, each of which had $1.6 \times 1.6 \mathrm{~mm}^{2}$ area. $y^{\prime}$ is the path length of incident $X$-rays in the phantom, while $x^{i}$ is the path length of XRF photons of the ith pixel in the phantom.

Abbreviation: XRF, X-ray fluorescence. 
where $N_{m}$ and $\mu_{m}$ are the number of photons and the linear attenuation coefficient of water at the photon energy $m$, respectively. $E_{K}$ is the energy right above the $K_{\text {edge }}$ energy (ie, Gd: $E_{K}=51 \mathrm{keV}$ and Au: $E_{K}=81 \mathrm{keV}$ ), while $E_{\max }$ is the maximum energy of the incident polychromatic $\mathrm{X}$-rays.

As the radial distance from the central pixel of the detector array increased, the detection sensitivity at the peripheral pixels suffered from the inverse distance-square. ${ }^{64}$ Such sensitivity correction factors were determined from empirical data obtained by additional MC simulations. A cylindrical virtual disk source of $5 \mathrm{~cm}$ in diameter and $5 \mathrm{~mm}$ in height, isotropically emitting photons of the same energy with $\mathrm{K}$-shell fluorescence X-rays at $43 \mathrm{keV}$ for $\mathrm{Gd}$ and $68 \mathrm{keV}$ for $\mathrm{Au}$, was located in a position of the midplane of water phantom (Figure S5). The sensitivity of the pinhole collimator was best for photons originating from the center of the virtual source, whereas photons from the periphery were much less detectable. The number of photons detected in each pixel of detector was normalized with respect to the value at the central pixel. Reciprocals of the values in each pixel represent the sensitivity correction factors. By multiplying all the correction factors with the raw signals at each pixel, corrected pixel values were obtained. The corrected values were directly plotted on a 2D map, representing the location and concentration of Gd or Au columns. The Gaussian filter was applied to decrease the noise. The contrast-to-noise ratio (CNR) is given by the following equation:

$$
\mathrm{CNR}=\left(\bar{C}_{R O I}-\bar{C}_{b g}\right) / \sigma_{b g}
$$

where $\bar{C}_{R O I}$ and $\bar{C}_{b g}$ are the mean pixel values in the region of interest (ROI) and the background region, respectively, which was used to evaluate the image quality for the incident X-ray spectra. The ROIs were the pixels projected by the $\mathrm{Gd}$ or $\mathrm{Au}$ columns, while the background region was set to the central pixels of field of view (FOV). $\sigma_{b g}$ is the standard deviation of the pixel values in the background region.

\section{Results}

\section{Geometric efficiency, system resolution, and energy resolution}

Since the energies of the XRF photons were relatively low, the effective pinhole diameter was almost similar to the physical pinhole diameter, which indicated no significant penetration effect at the edge of the aperture. Furthermore, the geometric efficiency, which depends on the angle $(\theta)$, was $1.65 \times 10^{-3}$ for the minimum angle (ie, $0^{\circ}$ ) and $5.03 \times 10^{-4}$ for the maximum angle (ie, $47.7^{\circ}$ ). The imaging system had the system resolution of $4.22 \mathrm{~mm}$, while the collimator and intrinsic resolutions were $3.90 \mathrm{~mm}$ and $1.60 \mathrm{~mm}$, respectively. From the measurement of the Am-241 photon peak at $59.5 \mathrm{keV}, \% \mathrm{FWHM}$ of the detector was determined to be $18.6 \%$ (ie, $11.1 \mathrm{keV}$ ) (Figure S2). According to this $\% \mathrm{FWHM}$, the original MC spectra of $1 \mathrm{keV}$ energy bin acquired by the polychromatic X-rays were degraded using the Gaussian distribution (MATLAB 2014a; MathWorks, Inc, Natick, MA, USA).

\section{Imaging of $\mathrm{Gd}$}

Figure 4 shows the 2D pinhole K-shell XRF images of $\mathrm{Gd}$ columns for $51 \mathrm{keV}$ and $110 \mathrm{kVp} \mathrm{X-rays.} \mathrm{The} \mathrm{subtracted}$ XRF images were obtained by subtracting the photon counts at the $41-46 \mathrm{keV}$ energy window of pure water phantom from those of Gd-loaded water phantom. The attenuation correction and the sensitivity correction were applied to the subtracted XRF images to generate corrected XRF images. Thus, $51 \mathrm{keV} \mathrm{X}$-rays induced higher XRF counts than $110 \mathrm{kVp}$ X-rays. Furthermore, even Gd columns with the lowest concentration at $0.01 \mathrm{wt} \%$ were still detectable in the corrected XRF images.

The linear relationship between the XRF signals and Gd concentrations was plotted, as shown in Figure 5A. Two curves for the incident X-rays showed a strong linearity (ie, $R^{2}>0.9827$ ), which implied that the corrections for the attenuation and sensitivity worked well. The CNRs of the Gd columns calculated from the pinhole K-shell XRF images are shown in Figure 5B. There was no significant difference in the CNRs of $51 \mathrm{keV}$ and $110 \mathrm{kVp}$ images.

\section{Imaging of $A u$}

The 2D pinhole K-shell XRF images of Au columns for $81 \mathrm{keV}$ and $140 \mathrm{kVp}$ X-rays are shown in Figure 6. The subtracted XRF images were obtained by subtracting the photon counts at the $66-71 \mathrm{keV}$ energy window of the pure water phantom from those of the Au-loaded water phantom. The attenuation and sensitivity corrections were applied to the subtracted XRF images to generate the corrected XRF images. The acquired images showed that more blurred regions were present for the images taken at $140 \mathrm{kVp}$ than for those taken at $81 \mathrm{keV}$. After the correction factors were applied to the subtracted $140 \mathrm{kVp}$ images, the blurred regions near the $0.06 \mathrm{wt} \% \mathrm{Au}$ became conspicuous.

A good linear relationship (ie, $R^{2}>0.9162$ ) was established between the integrated XRF signals and the Au concentrations, as shown in Figure 7A. The CNRs of the $81 \mathrm{keV}$ and $140 \mathrm{kVp}$ images were similar, except for $0.06 \mathrm{wt} \% \mathrm{Au}$ 

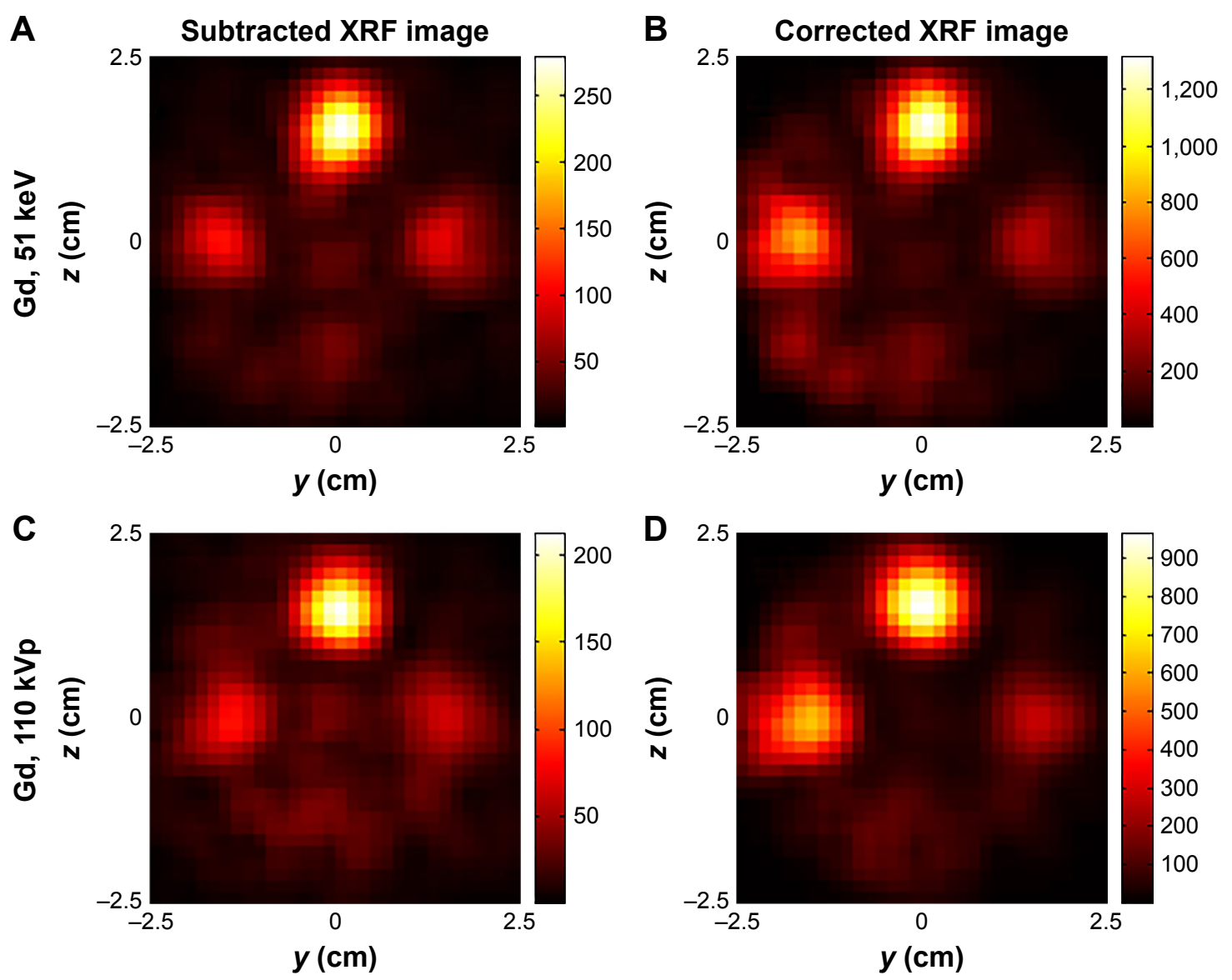

Figure 4 Subtracted and corrected XRF images for Gd.

Notes: (A) Subtracted XRF image between Gd-loaded water phantom and pure water phantom; (B) XRF image of Gd corrected by attenuation and sensitivity for monochromatic 5 I keV X-rays; (C) subtracted XRF image between Gd-loaded water phantom and pure water phantom; and (D) XRF image of Gd corrected by attenuation and sensitivity for polychromatic $110 \mathrm{kVp}$ X-rays.

Abbreviations: XRF, X-ray fluorescence; Gd, gadolinium.

(Figure 7B). For the $0.01 \mathrm{wt} \% \mathrm{Au}$ columns, the CNRs of $0.01 \mathrm{wt} \% \mathrm{Au}$ columns were 0.7 and 1.0 for $81 \mathrm{keV}$ and $140 \mathrm{kVp}$ images, respectively. For $140 \mathrm{kVp}$ X-rays, according to the Rose criterion for CNR, the lowest distinguishable concentrations were $0.03 \mathrm{wt} \%$ for the $140 \mathrm{kVp}$ images.

\section{Radiation dose}

The imaging doses of the pinhole XRF imaging system are summarized in Table 1. These radiation doses were calculated with $1 \times 10^{11}$ histories of incident $X$-rays, which was also the number of histories to acquire all the images shown before.
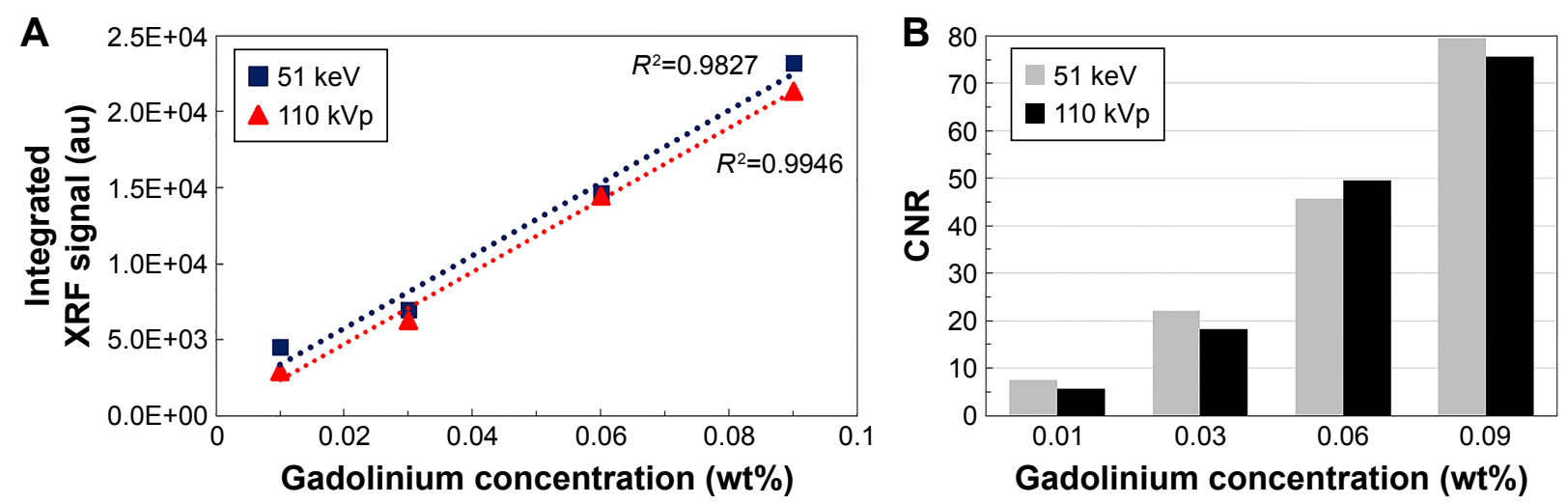

Figure 5 XRF signal intensities and CNR values versus Gd concentrations.

Notes: (A) Linear relationship between Gd concentrations and integrated XRF counts and (B) CNR values in the region of Gd columns for two different X-ray source spectra. Abbreviations: CNR, contrast-to-noise ratio; XRF, X-ray fluorescence; wt, weight; Gd, gadolinium. 
A

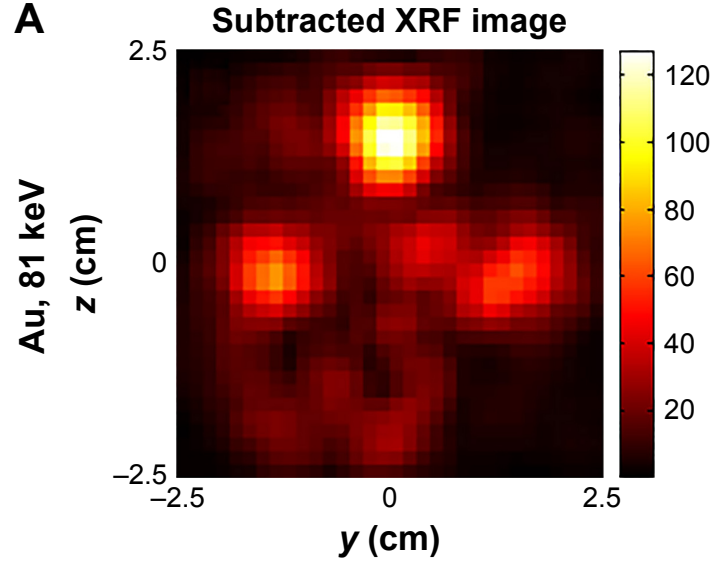

C

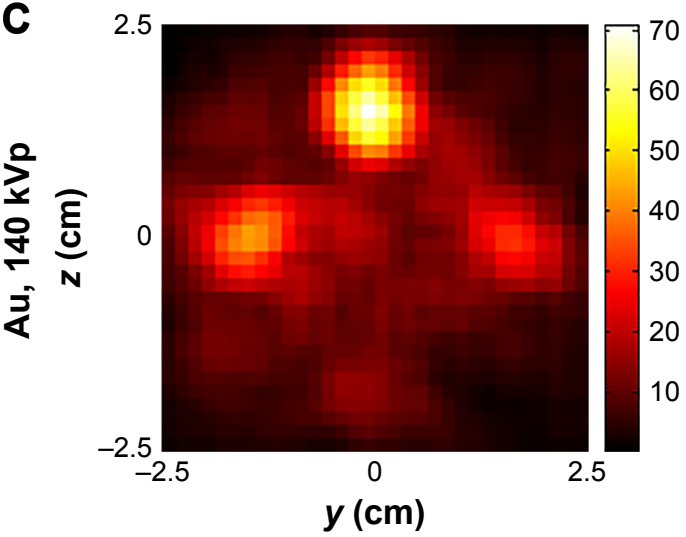

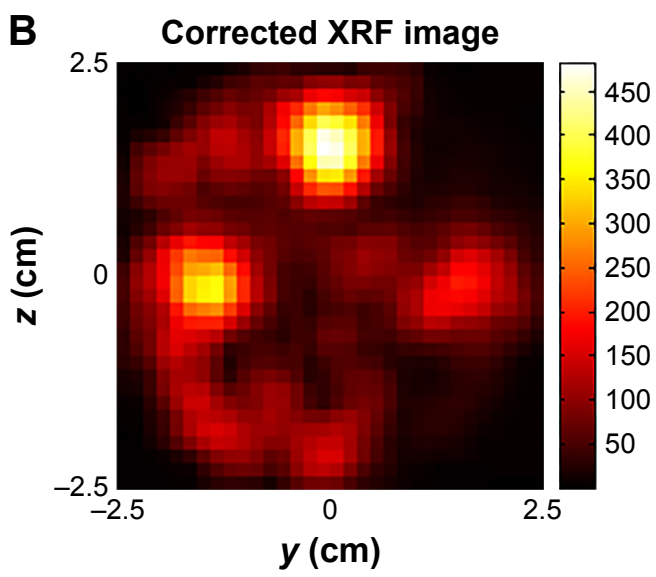

D

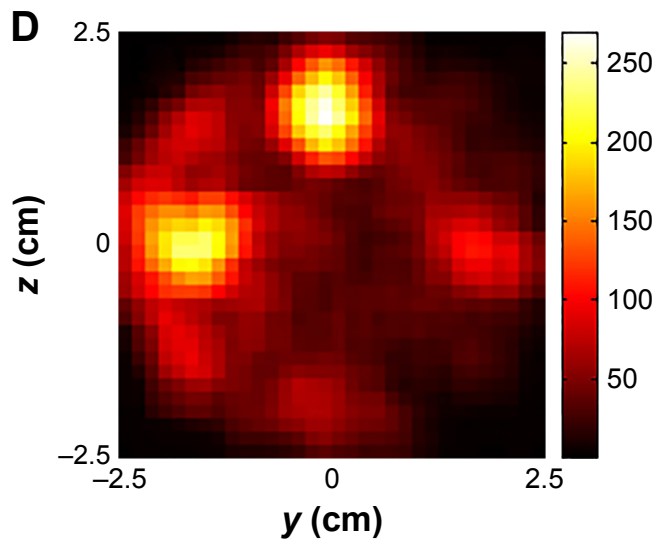

Figure 6 Subtracted and corrected XRF images for Au.

Notes: (A) Subtracted XRF image between Au-loaded water phantom and pure water phantom; (B) XRF image of Au corrected by attenuation and sensitivity for monochromatic $81 \mathrm{keV} X$-rays; (C) subtracted XRF image between Au-loaded water phantom and pure water phantom; and (D) XRF image of Au corrected by attenuation and sensitivity for polychromatic $140 \mathrm{kV} p$ X-rays.

Abbreviations: XRF, X-ray fluorescence; Au, gold.

The imaging dose was composed of two components, the prescanning dose of the phantom without $\mathrm{Gd}$ or $\mathrm{Au}$ and the XRF scanning dose of the phantom with Gd or Au. The radiation doses with NP in the phantom were a little higher (by $0.1 \mathrm{mGy}$ ) than the dose for the pure water phantom. The total imaging dose is the sum of these two components.

\section{Discussion}

Pinhole K-shell XRF imaging using polychromatic X-rays and $2 \mathrm{D}$ array of position-sensitive detectors appears to be feasible for in vivo imaging and quantification of GdNP- or AuNP-loaded columns within small-animal-sized phantoms. The developed model can acquire 2D XRF images directly
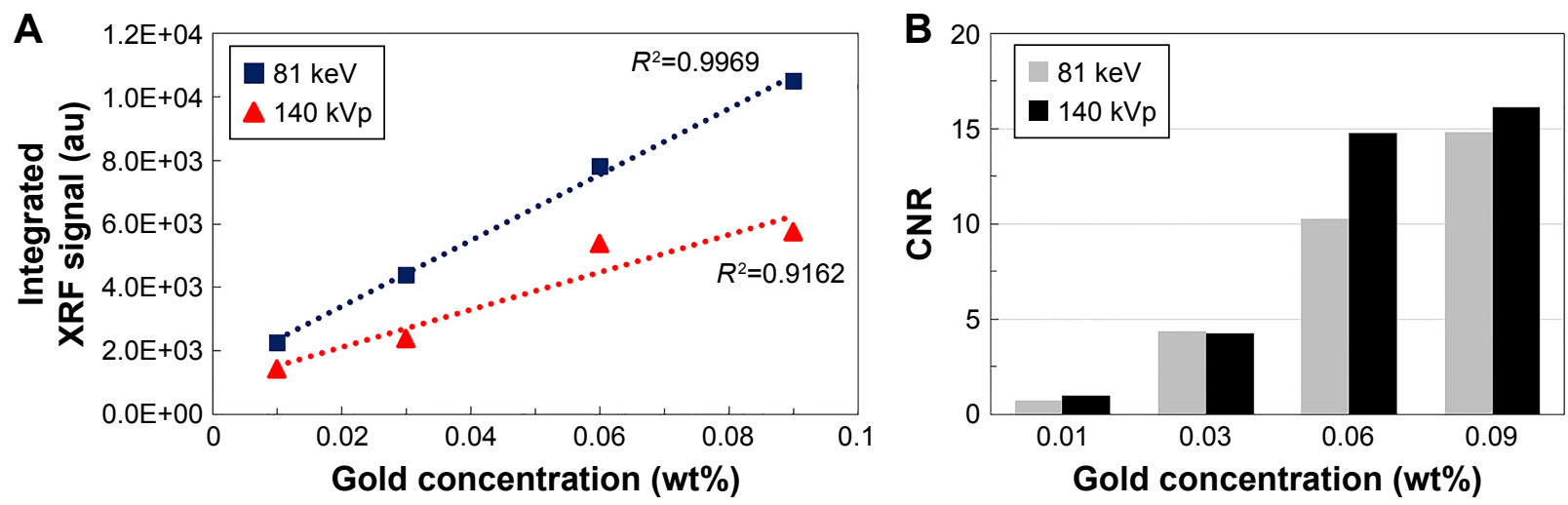

Figure 7 XRF signal intensities and CNR values versus Au concentrations.

Note: (A) Linear relationship between Au concentrations and integrated XRF counts and (B) CNR values in the region of Au columns for two different X-ray source spectra. Abbreviations: CNR, contrast-to-noise ratio; XRF, X-ray fluorescence; Au, gold. 
Table I Imaging dose of pinhole XRF imaging for four different incident X-ray sources

\begin{tabular}{llll}
\hline Incident & \multicolumn{2}{l}{ Average dose, $\mathbf{m G y}$} \\
\cline { 2 - 4 } X-rays & Pure water & NP-loaded water phantom & Sum \\
\hline $51 \mathrm{keV}$ & 10.1 & 10.2 & 20.3 \\
$110 \mathrm{kV} P$ & 9.9 & 10.0 & 19.9 \\
$81 \mathrm{keV}$ & 10.6 & 10.7 & 21.3 \\
$140 \mathrm{kVP}$ & 13.0 & 13.1 & 26.1 \\
\hline
\end{tabular}

Abbreviations: NP, nanoparticle; XRF, X-ray fluorescence.

from only one fan-beam irradiation without the need of further processing like beam or detector translation and phantom rotation.

Although $51 \mathrm{keV}$ and $81 \mathrm{keV} \mathrm{X-rays} \mathrm{are} \mathrm{close} \mathrm{to} \mathrm{the} K_{\text {edge }}$ energies of $\mathrm{Gd}$ and $\mathrm{Au}$, respectively, the resulting images obtained by $110 \mathrm{kVp}$ and $140 \mathrm{kVp}$ beams showed similar level of image quality. Blurred regions in XRF images of the polychromatic X-rays were caused by the statistical noise from the subtraction of the Compton scatter background, which was sometimes larger than the signals of XRF. Although their integrated XRF counts were less than those of the monochromic X-rays, the proposed polychromatic X-rays could generate recognizable K-shell XRF images for $0.01 \mathrm{wt} \% \mathrm{GdNP}$ and $0.03 \mathrm{wt} \%$ AuNP. For Au imaging, $140 \mathrm{kVp}$ showed higher CNR in $0.06 \mathrm{wt} \%$ Au than that in the same region for the $81 \mathrm{keV}$ XRF image. This might be caused by a slight overestimation of the attenuation correction factors, especially for deep-seated regions (ie, $0.06 \mathrm{wt} \% \mathrm{Au}$ ). The use of polychromatic X-rays showed blurring in the $\mathrm{Gd}$ or Au images because polychromatic $\mathrm{X}$-rays generated less $\mathrm{XRF}$ photons than monochromatic X-rays. Moreover, the false photon counts from the scattered polychromatic X-rays in the phantom may have contributed to the blurring.

In the MC simulation, the images were degraded by the measured \%FWHM. In fact, the radiation transport codes could not solely include the physical degradations of the detector response such as electron-hole trapping, nonuniformity, and charge sharing ${ }^{65-67}$ Therefore, the \%FWHM of the CZT detector was measured to overcome the limitation of the MC study. Since the measured \%FWHM applied to the simulated spectra was pixel-averaged, there is a possible overestimation in the quality of the processed images. In addition, any systematic errors could not be explicitly considered in this MC study. In order to reduce the interscanning systematic errors (eg, positioning and environmental condition for CZT detector), the experimental setup should be coincident for both prescanning and XRF scanning.

The current imaging system using commercial pixelated CZT gamma cameras has limitations in terms of system resolution (eg, pixel size and pinhole diameter) and energy resolution. Use of a smaller detector pixel or a pinhole is possible and is expected to improve the system resolution. However, these modifications would dramatically reduce the photon counts per pixel, which would result in considerable increase in the imaging dose and irradiation time. A compromise between system resolution and irradiation time is required. There have been technical efforts to improve the energy resolution of pixelated semiconductor detectors. ${ }^{66} \mathrm{Such}$ detectors have been manufactured by several research groups for the purpose of detecting gamma rays in space ${ }^{66,68}$ but are yet to be implemented for medical imaging purposes.

The previous XFCT system using a single CdTe detector took 90 minutes to get a single 2D slice image of a mouse. ${ }^{69}$ As proposed in this study, if a 2D array of position-sensitive detectors can measure XRF and scattered photons, the image acquisition time can be dramatically reduced. Compared with the parallel-hole collimator XFCT system, the pinhole XRF imaging suffers from low detection efficiency. However, the pinhole system does not need to translate and rotate the sample to acquire a 2D slice image as required for the parallel-hole system. Moreover, the system can offer magnified images by simply adjusting the object-to-pinhole and pinhole-todetector distances. Meng et $\mathrm{al}^{45}$ and $\mathrm{Fu}$ et $\mathrm{al}^{46}$ also demonstrated that the pinhole imaging system could detect more XRF photons per unit time and that the extended volume of phantom irradiated by the incident X-rays improved the imaging speed. Therefore, these advantages could offset the low detection efficiency of the pinhole system. Furthermore, implementation of the multipinhole collimation system may improve the imaging speed and efficiency.

However, the dose level ( $\sim 30 \mathrm{mGy})$ predicted by MC simulations of our imaging system was much less than the imaging doses (a few hundred mGy) delivered by XFCT and micro-CT scanning of small animals. ${ }^{69,70}$ Since our system was modeled by benchmarking the pinhole SPECT system, which detects much higher energy photons than K-shell XRF photons ${ }^{52-56}$ further investigation in optimizing the system design - such as collimator design, X-ray filter, and tube potential - is needed to reduce the dose level and improve the image quality. In addition, if the aforementioned 2D pixelated detectors with high-energy resolution are commercially available, discrete XRF peaks can be analytically separated from the background photons. This eliminates the prescanning procedure and, thus, can reduce the imaging dose as well as the systematic errors by half.

\section{Conclusion}

The MC simulations demonstrated the feasibility of the pinhole K-shell XRF imaging system for GdNPs and AuNPs stimulated 
by polychromatic X-rays using a 2D position-sensitive array of CZT pixelated detectors. The MC model for this pinhole XRF system showed direct 2D slice images of the object without image reconstruction. In a cylindrical water phantom of $5 \mathrm{~cm}$ depth and height, $0.01 \mathrm{wt} \%$ of GdNP and $0.03 \mathrm{wt} \%$ of AuNP were shown to be the minimum concentrations to achieve the Rose criterion for CNR $(\mathrm{CNR}=4)$. The $\mathrm{MC}$ technique developed in this study will be used to optimize experimental setups for future developments of the pinhole XRF imaging system.

\section{Acknowledgments}

This work was supported by the National Research Foundation of Korea (NRF) grant (number NRF-2013M2B2B1075776) funded by the Korean government (Ministry of Science, ICT and Future Planning). Part of the results of this study have been presented at the Korean Nuclear Society (KNS) 2014 Fall Meeting and the 2015 Institute of Electrical and Electronics Engineers (IEEE) Nuclear Science Symposium and Medical Imaging Conference. The conference record was published in the proceedings of the conference (2015 IEEE NSS/MIC).

\section{Author contributions}

SJY conceived the study concept, participated in all aspects of its design and coordination, and helped draft the manuscript. SJ performed MC simulations and drafted the manuscript. WS managed and commented on the methods for simulations and analysis. All authors contributed toward data analysis, drafting and critically revising the paper, gave final approval of the version to be published, and agree to be accountable for all aspects of the work.

\section{Disclosure}

The authors report no conflicts of interest in this work.

\section{References}

1. Salata O. Applications of nanoparticles in biology and medicine. J Nanobiotechnology. 2004;2(1):3.

2. De Jong WH, Borm PJ. Drug delivery and nanoparticles: applications and hazards. Int J Nanomedicine. 2008;3(2):133-149.

3. Jain PK, Huang X, El-Sayed IH, El-Sayed MA. Noble metals on the nanoscale: optical and photothermal properties and some applications in imaging, sensing, biology, and medicine. Acc Chem Res. 2008;41(12): $1578-1586$.

4. Zhang L, Gu FX, Chan JM, Wang AZ, Langer RS, Farokhzad OC. Nanoparticles in medicine: Therapeutic applications and developments. Clin Pharmacol Ther. 2008;83(5):761-769.

5. Nazir S, Hussain T, Ayub A, Rashid U, MacRobert AJ. Nanomaterials in combating cancer: therapeutic applications and developments. Nanomedicine. 2014;10(1):19-34.

6. Paunesku T, Ke T, Dharmakumar R, et al. Gadolinium-conjugated $\mathrm{TiO}_{2}$-DNA oligonucleotide nanoconjugates show prolonged intracellular retention period and T1-weighted contrast enhancement in magnetic resonance images. Nanomedicine. 2008;4(3):201-207.
7. Park JY, Baek MJ, Choi ES, et al. Paramagnetic ultrasmall gadolinium oxide nanoparticles as advanced T1 MRI contrast agent: account for large longitudinal relaxivity, optimal particle diameter, and in vivo T1 MR images. ACS Nano. 2009;3(11):3663-3669.

8. Kim TJ, Chae KS, Chang Y, Lee GH. Gadolinium oxide nanoparticles as potential multimodal imaging and therapeutic agents. Curr Top Med Chem. 2013;13(4):422-433.

9. Porcel E, Tillement O, Lux F, et al. Gadolinium-based nanoparticles to improve the hadron therapy performances. Nanomedicine. 2014; 10(8):1601-1608.

10. Schlatholter T, Eustache P, Porcel E, et al. Improving proton therapy by metal-containing nanoparticles: nanoscale insights. Int J Nanomedicine. 2016;11:1549-1556.

11. Taupin F, Flaender M, Delorme R, et al. Gadolinium nanoparticles and contrast agent as radiation sensitizers. Phys Med Biol. 2015;60(11): 4449-4464.

12. Khan AK, Rashid R, Murtaza G, Zahra A. Gold nanoparticles: synthesis and applications in drug delivery. Trop J Pharm Res. 2014;13(7): 1169-1177.

13. Hainfeld JF, Slatkin DN, Smilowitz HM. The use of gold nanoparticles to enhance radiotherapy in mice. Phys Med Biol. 2004;49(18): N309-N315.

14. Cho SH, Jones BL, Krishnan S. The dosimetric feasibility of gold nanoparticle-aided radiation therapy (GNRT) via brachytherapy using low-energy gamma-/X-ray sources. Phys Med Biol. 2009;54(16): 4889-4905.

15. Rahman WN, Bishara N, Ackerly T, et al. Enhancement of radiation effects by gold nanoparticles for superficial radiation therapy. Nanomedicine. 2009;5(2):136-142.

16. Kim JK, Seo SJ, Kim HT, et al. Enhanced proton treatment in mouse tumors through proton irradiated nanoradiator effects on metallic nanoparticles. Phys Med Biol. 2012;57(24):8309-8323.

17. Ngwa W, Korideck H, Kassis AI, et al. In vitro radiosensitization by gold nanoparticles during continuous low-dose-rate gamma irradiation with I-125 brachytherapy seeds. Nanomedicine. 2013;9(1): 25-27.

18. Lin Y, McMahon SJ, Paganetti H, Schuemann J. Biological modeling of gold nanoparticle enhanced radiotherapy for proton therapy. Phys Med Biol. 2015;60(10):4149-4168.

19. Paro AD, Hossain M, Webster TJ, Su M. Monte Carlo and analytic simulations in nanoparticle-enhanced radiation therapy. Int $J$ Nanomedicine. 2016;11:4735-4741.

20. Sung W, Jung S, Ye SJ. Evaluation of the microscopic dose enhancement for nanoparticle-enhanced Auger therapy. Phys Med Biol. 2016; 61(21):7522-7535.

21. Liu Y, Liu X, Jin X, et al. The dependence of radiation enhancement effect on the concentration of gold nanoparticles exposed to low- and high-LET radiations. Phys Med. 2015;31(3):210-218.

22. Her S, Jaffray DA, Allen C. Gold nanoparticles for applications in cancer radiotherapy: mechanisms and recent advancements. Adv Drug Deliv Rev. 2017;109:84-101.

23. Schuemann J, Berbeco R, Chithrani DB, et al. Roadmap to clinical use of gold nanoparticles for radiation sensitization. Int J Radiat Oncol Biol Phys. 2016;94(1):189-205.

24. Subiel A, Ashmore R, Schettino G. Standards and methodologies for characterizing radiobiological impact of high- $Z$ nanoparticles. Theranostics. 2016;6(10):1651-1671.

25. Sung W, Ye SJ, McNamara AL, et al. Dependence of gold nanoparticle radiosensitization on cell geometry. Nanoscale. 2017;9(18) $5843-5853$.

26. Roa W, Zhang XJ, Guo LH, et al. Gold nanoparticle sensitize radiotherapy of prostate cancer cells by regulation of the cell cycle. Nanotechnology. 2009;20(37):375101.

27. Chithrani DB, Jelveh S, Jalali F, et al. Gold nanoparticles as radiation sensitizers in cancer therapy. Radiat Res. 2010;173(6):719-728.

28. Kang B, Mackey MA, El-Sayed MA. Nuclear targeting of gold nanoparticles in cancer cells induces DNA damage, causing cytokinesis arrest and apoptosis. $J$ Am Chem Soc. 2010;132(5):1517. 
29. Jain S, Coulter JA, Hounsell AR, et al. Cell-specific radiosensitization by gold nanoparticles at megavoltage radiation energies. Int $J$ Radiat Oncol Biol Phys. 2011;79(2):531-539.

30. Mackey MA, Saira F, Mahmoud MA, El-Sayed MA. Inducing cancer cell death by targeting its nucleus: solid gold nanospheres versus hollow gold nanocages. Bioconjug Chem. 2013;24(6):897-906.

31. Cui L, Tse K, Zahedi P, et al. Hypoxia and cellular localization influence the radiosensitizing effect of gold nanoparticles (AuNPs) in breast cancer cells. Radiat Res. 2014;182(5):475-488.

32. Chithrani BD, Ghazani AA, Chan WCW. Determining the size and shape dependence of gold nanoparticle uptake into mammalian cells. Nano Lett. 2006;6(4):662-668.

33. Lee SY, Ferrari M, Decuzzi P. Shaping nano-/micro-particles for enhanced vascular interaction in laminar flows. Nanotechnology. 2009; 20(49):495101.

34. Butterworth KT, Coulter JA, Jain S, et al. Evaluation of cytotoxicity and radiation enhancement using $1.9 \mathrm{~nm}$ gold particles: potential application for cancer therapy. Nanotechnology. 2010;21(29):295101.

35. Arnida, Janat-Amsbury MM, Ray A, et al. Geometry and surface characteristics of gold nanoparticles influence their biodistribution and uptake by macrophages. Eur J Pharm Biopharm. 2011;77:417-423.

36. Huang KY, Ma HL, Liu J, et al. Size-dependent localization and penetration of ultrasmall gold nanoparticles in cancer cells, multicellular spheroids, and tumors in vivo. ACS Nano. 2012;6(5):4483-4493.

37. Cho SH, Krishnan S. Cancer Nanotechnology: Principles and Applications in Radiation Oncology. Boca Raton, FL: CRC Press; 2013.

38. Cheong SK, Jones BL, Siddiqi AK, Liu F, Manohar N, Cho SH. X-ray fluorescence computed tomography (XFCT) imaging of gold nanoparticle-loaded objects using $110 \mathrm{kVp} \mathrm{X-rays.} \mathrm{Phys} \mathrm{Med} \mathrm{Biol.}$ 2010;55(3):647-662.

39. Boisseau P. Determination of Three Dimensional Trace Element Distributions by the Use of Monochromatic X-Ray Microbeams [Ph.D. dissertation]. Cambridge, MA: Massachusetts Institute of Technology; 1986.

40. Jones BL, Manohar N, Reynoso F, Karellas A, Cho SH. Experimental demonstration of benchtop X-ray fluorescence computed tomography (XFCT) of gold nanoparticle-loaded objects using lead- and tin-filtered polychromatic cone-beams. Phys Med Biol. 2012;57(23): N457-N467.

41. Ahmad M, Bazalova-Carter M, Fahrig R, Xing L. Optimized detector angular configuration increases the sensitivity of X-ray fluorescence computed tomography (XFCT). IEEE Trans Med Imaging. 2015;34(5): $1140-1147$

42. Manohar N, Reynoso FJ, Cho SH. Experimental demonstration of direct L-shell X-ray fluorescence imaging of gold nanoparticles using a benchtop X-ray source. Med Phys. 2013;40(8):080702.

43. Bazalova-CarterM. The potential of L-shell X-ray fluorescenceCT(XFCT) for molecular imaging. Br J Radiol. 2015;88(1055):20140308.

44. Bazalova-Carter M, Ahmad M, Xing L, Fahrig R. Experimental validation of L-shell X-ray fluorescence computed tomography imaging: phantom study. J Med Imaging (Bellingham). 2015;2(4):043501.

45. Meng LJ, Li N, La Riviere PJ. X-ray fluorescence emission tomography (XFET) with novel imaging geometries - a Monte Carlo study. IEEE Trans Nucl Sci. 2011;58(6):3359-3369.

46. Fu G, Meng LJ, Eng P, Newville M, Vargas P, La Riviere P. Experimental demonstration of novel imaging geometries for X-ray fluorescence computed tomography. Med Phys. 2013;40(6):061903.

47. Groll A, George J, Vargas P, La Riviere PJ, Meng LJ. Element mapping in organic samples utilizing a benchtop X-ray fluorescence emission tomography (XFET) system. IEEE Trans Nucl Sci. 2015;62(5): 2310-2317.

48. Goorley T, James M, Booth T, et al. Initial Mcnp6 release overview. Nucl Technol. 2012;180(3):298-315.

49. Alnafea M, Wells K, Spyrou NM, Guy M. Preliminary Monte Carlo study of coded aperture imaging with a CZT gamma camera system for scintimammography. Nucl Instrum Meth A. 2007;573(1-2): $122-125$.
50. Campbell DL, Peterson TE. Simulation study comparing high-purity germanium and cadmium zinc telluride detectors for breast imaging. Phys Med Biol. 2014;59(22):7059-7079.

51. Manohar N, Jones BL, Cho SH. Improving X-ray fluorescence signal for benchtop polychromatic cone-beam X-ray fluorescence computed tomography by incident X-ray spectrum optimization: a Monte Carlo study. Med Phys. 2014;41(10):101906.

52. Jaszczak RJ, Li JY, Wang HL, Zalutsky MR, Coleman RE. Pinhole collimation for ultra-high-resolution, small-field-of-view SPECT. Phys Med Biol. 1994;39(3):425-437.

53. Cherry SR, Sorenson JA, Phelps ME. Physics in Nuclear Medicine. 3rd ed. Philadelphia, PA: Saunders; 2003.

54. Deloar HM, Watabe H, Aoi T, Iida H. Evaluation of penetration and scattering components in conventional pinhole SPECT: phantom studies using Monte Carlo simulation. Phys Med Biol. 2003;48(8):995-1008.

55. Moji V, Zeraatkar N, Farahani MH, et al. Performance evaluation of a newly developed high-resolution, dual-head animal SPECT system based on the NEMA NU1-2007 standard. J Appl Clin Med Phys. 2014; 15(6):267-278.

56. Peterson M, Strand SE, Ljungberg M. Using Rose's metal alloy as a pinhole collimator material in preclinical small-animal imaging: a Monte Carlo evaluation. Med Phys. 2015;42(4):1698-1709.

57. Iwanczyk JS, Iniewski K. Radiation Detectors for Medical Imaging. Boca Raton, FL: CRC Press; 2016.

58. Poludniowski G, Landry G, DeBlois F, Evans PM, Verhaegen F. SpekCalc: a program to calculate photon spectra from tungsten anode X-ray tubes. Phys Med Biol. 2009;54(19):N433-N438.

59. Pidikiti R, Stojadinovic S, Speiser M, et al. Dosimetric characterization of an image-guided stereotactic small animal irradiator. Phys Med Biol. 2011;56(8):2585-2599.

60. Mesbahi A, Zakariaee SS. Effect of anode angle on photon beam spectra and depth dose characteristics for X-RAD320 orthovoltage unit. Rep Pract Oncol Radiother. 2013;18(3):148-152.

61. Noblet C, Chiavassa S, Smekens F, et al. Validation of fast Monte Carlo dose calculation in small animal radiotherapy with EBT3 radiochromic films. Phys Med Biol. 2016;61(9):3521-3535.

62. Perkins ST, Cullen DE, Chen MH, Hubbell JH, Rathkopf J, Scofield J. Tables and graphs of atomic subshell and relaxation data derived from the LLNL Evaluated Atomic Data Library (EADL), Z=1-100. Lawrence Livermore National Laboratory, Report UCRL-50400 Series, Livermore, CA; 1991.

63. Hubbell JH, Seltzer SM. Tables of X-ray mass attenuation coefficients and mass energy-absorption coefficients $1 \mathrm{keV}$ to $20 \mathrm{MeV}$ for elements $\mathrm{Z}=1$ to 92 and 48 additional substances of dosimetric interest. National Inst. Of Standards and Technology-PL, Gaithersburg, MD, Technical Report, TRN:51812148 1995.

64. Connolly LP, Treves ST, Davis RT, Zimmerman RE. Pediatric applications of pinhole magnification imaging. J Nucl Med. 1999; 40(11):1896-1901.

65. d'Aillon EG, Tabary J, Gliere A, Verger L. Charge sharing on monolithic CdZnTe gamma-ray detectors: a simulation study. Nucl Instrum Meth A. 2006;563(1):124-127.

66. Del Sordo S, Abbene L, Caroli E, Mancini AM, Zappettini A, Ubertini P. Progress in the development of CdTe and CdZnTe semiconductor radiation detectors for astrophysical and medical applications. Sensors (Basel). 2009;9(5):3491-3526.

67. Bolotnikov AE, Camarda GS, Cui Y, et al. Characterization and evaluation of extended defects in CZT crystals for gamma-ray detectors. J Cryst Growth. 2013;379:46-56.

68. KoKubun M, Nakazawa K, Enoto T, et al. Hard X-ray imager (HXI) for the ASTRO-H mission. Proc SPIE. 2010;7732:773215.

69. Manohar N, Reynoso FJ, Diagaradjane P, Krishnan S, Cho SH. Quantitative imaging of gold nanoparticle distribution in a tumor-bearing mouse using benchtop X-ray fluorescence computed tomography. Sci Rep. 2016;6:22079.

70. Boone JM, Velazquez O, Cherry SR. Small-animal X-ray dose from micro-CT. Mol Imag. 2004;3(3):149-158. 


\section{Supplementary materials Geometric efficiency (g)}

The geometric efficiency $(g)$ was calculated as follows: ${ }^{1}$

$$
\begin{gathered}
g \approx d_{e f f} \cos ^{3} \theta / 16 b^{2} \\
d_{e f f}=\sqrt{d\left[d+2 \mu^{-1} \tan (\alpha / 2)\right]}
\end{gathered}
$$

where $d_{\text {eff }}$ is the effective pinhole diameter, $\theta$ is the angle between the source (in our system, a midplane in the water phantom where the incident X-rays pass through) and a pinhole (Figure $\mathrm{S} 1$ ), and $b$ is the beam plane-to-pinhole distance. The effective pinhole diameter $d_{\text {eff }}$ accounts for penetration of the edges of the pinhole aperture.

\section{System resolution}

The system resolution was calculated as follows: ${ }^{1}$

$$
R_{\text {sys }}=\sqrt{R_{\text {int }}^{2}+R_{\text {coll }}^{2}}
$$

$$
R_{\text {coll }} \approx d_{\text {eff }}(l+b) / l
$$

where $R_{\text {int }}$ is the intrinsic resolution of the detector, $R_{\text {coll }}$ is the collimator resolution, and $l$ is the distance from the pinhole to the active surface of the detector. The field of view (FOV) in this configuration was $5 \mathrm{~cm}$ diameter; however, the magnification factor and FOV were adjustable by varying the beam plane-to-collimator and the collimator-to-detector distances.

\section{Percentage full width at half maximum (\%FWHM) measurement of Am-24I source with CZT gamma camera}

The Am-241 source was electrodeposited, resulting in an active area of $3 \mathrm{~mm}$ diameter on a palladium disk of $12.7 \mathrm{~mm}$ diameter and $0.127 \mathrm{~mm}$ thickness. At the source-to-detector distance of $5 \mathrm{~cm}$, the spectrum was measured by the pixelated CZT gamma camera for 1 hour at room temperature. All 1,024 pixel counts were averaged over to determine the $\%$ FWHM of the detector at $59.5 \mathrm{keV}$.

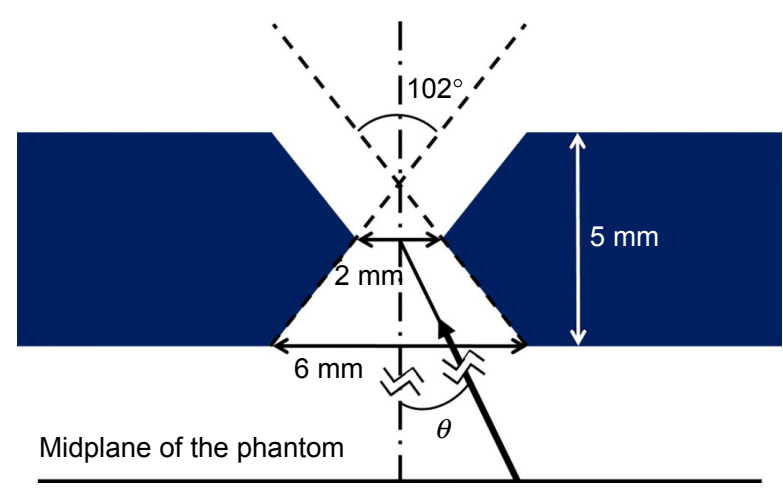

Figure SI Schematic of pinhole collimation, where the acceptance angle is $102^{\circ}$ and the angle between a point on the midplane of water phantom and the center of pinhole is $\theta$.

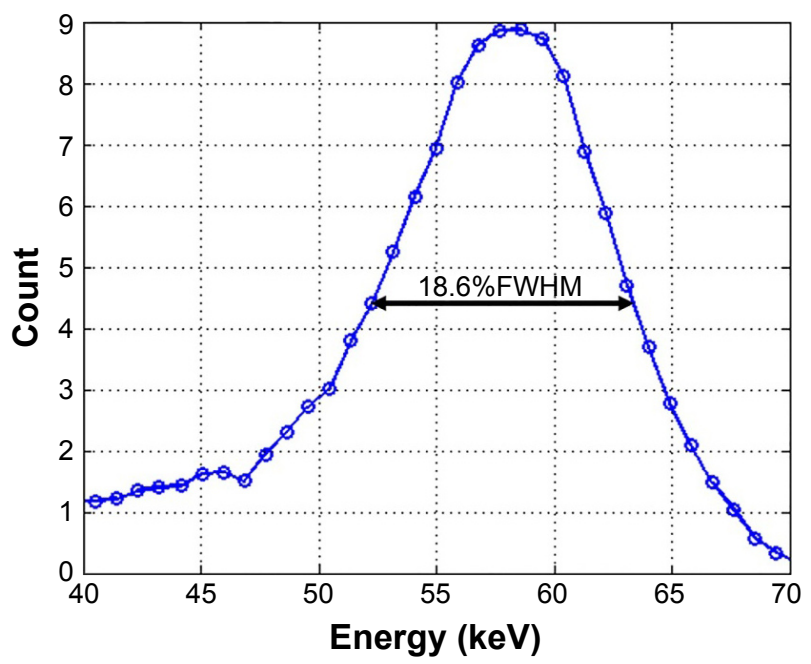

Figure S2 Energy spectrum of Am-24I acquired with the CZT gamma camera.

Abbreviations: $\mathrm{CZT}$, cadmium-zinc-telluride; FWHM, full width at half maximum. 


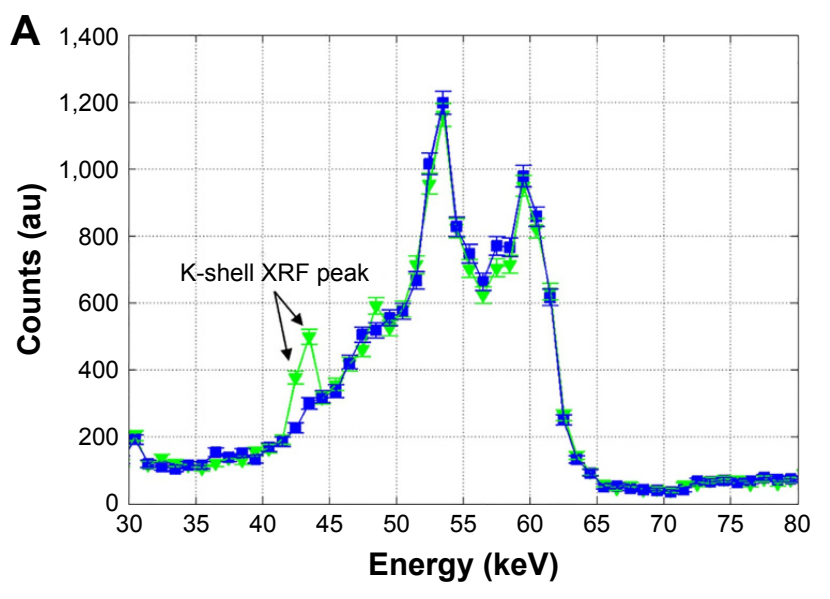

$\checkmark 110 \mathrm{kVp} \mathrm{Gd}+$ water (1 keV bin)

- $110 \mathrm{kVp}$ pure water (1 keV bin)

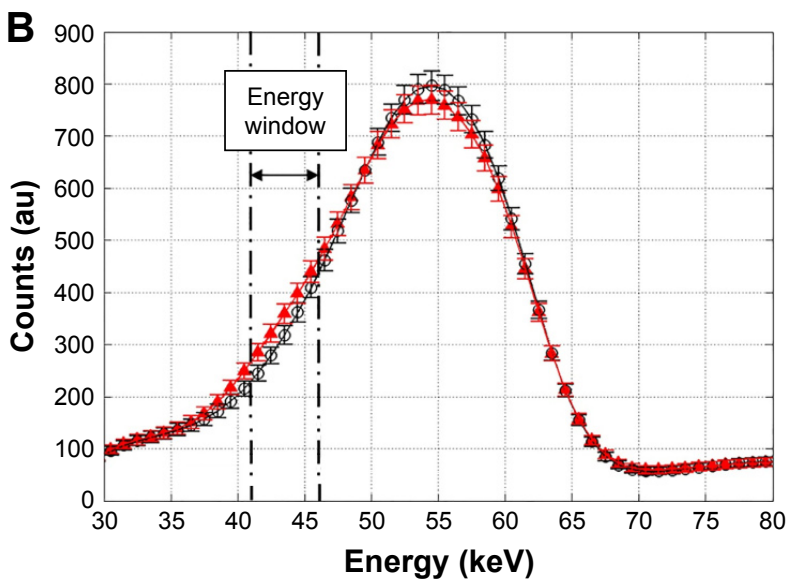

- $110 \mathrm{kVp} \mathrm{Gd}+$ water (degradation)

๑ $110 \mathrm{kVp}$ pure water (degradation)

Figure S3 Energy spectra of K-shell XRF and Compton scattered photons from 0.09 wt\% Gd column.

Notes: (A) Energy spectra of MC-based I keV bin and (B) energy spectra of a degradation with the measured \%FWHM. Error bar indicates $68 \%$ confidence level. Abbreviations: FWHM, full width at half maximum; MC, Monte Carlo; XRF, X-ray fluorescence; Gd, gadolinium.

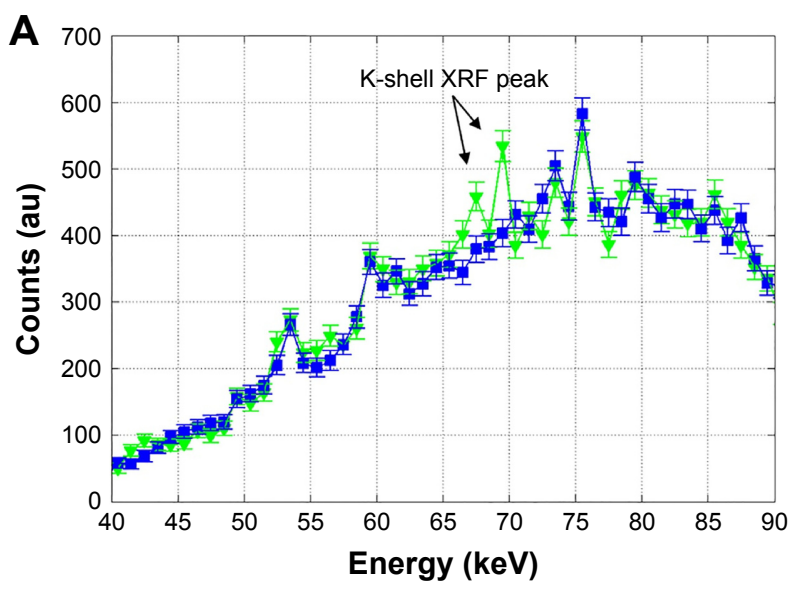

$-140 \mathrm{kVp} A u+$ water $(1 \mathrm{keV}$ bin)

- $140 \mathrm{kVp}$ pure water (1 keV bin)

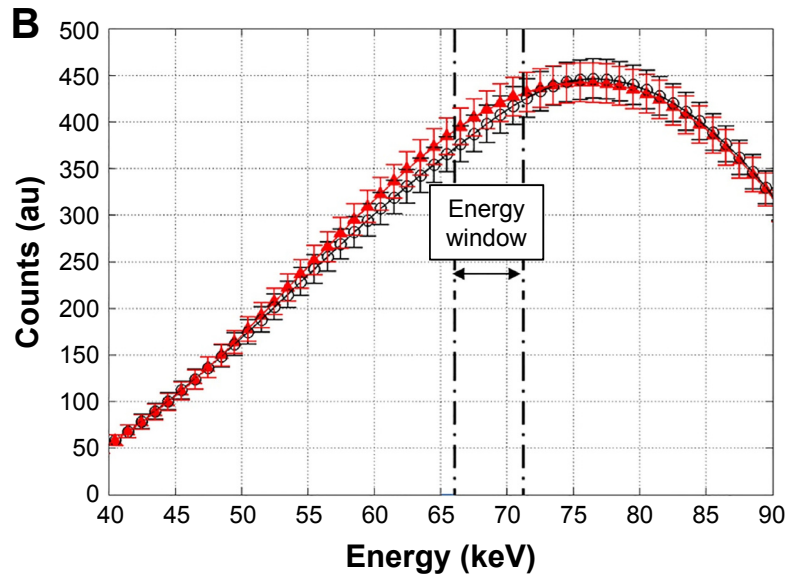

- $140 \mathrm{kVp} \mathrm{Au}+$ water (degradation) - $140 \mathrm{kVp}$ pure water (degradation)

Figure S4 Energy spectra of K-shell XRF and Compton scattered photons from 0.09 wt\% Au column.

Notes: (A) Energy spectra of MC-based I keV bin and (B) energy spectra of a degradation with the measured \%FWHM. Error bar indicates $68 \%$ confidence level.

Abbreviations: FWHM, full width at half maximum; MC, Monte Carlo; XRF, X-ray fluorescence; Au, gold; wt, weight. 


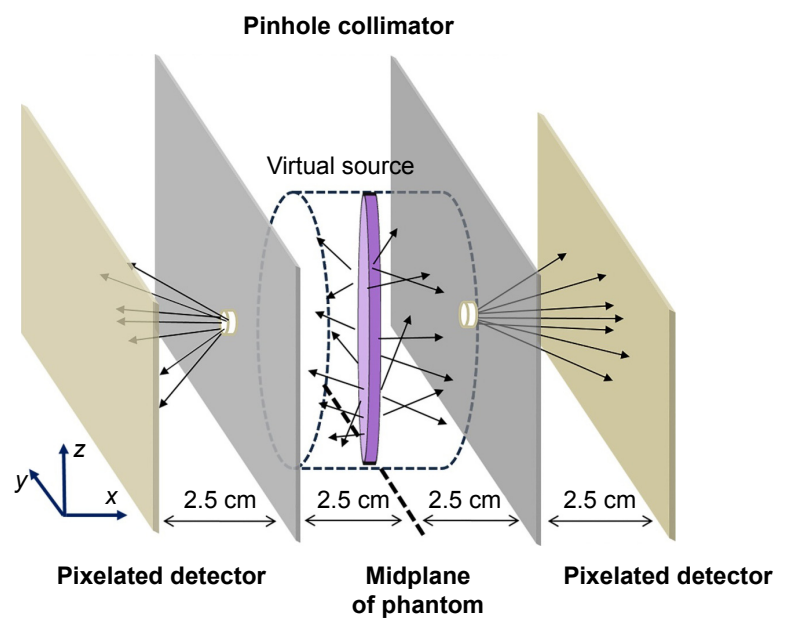

Figure S5 $M C$ simulation model for sensitivity correction.

Notes: The virtual disk source of $5 \mathrm{~cm}$ diameter and $5 \mathrm{~mm}$ width (purple) in the midplane of water phantom isotropically emits fluorescence-like photons. The arrows describe photons emitting from the virtual source. The photons originating from the center of the source are detected more efficiently than those from the periphery. Abbreviation: MC, Monte Carlo.

\section{Reference}

1. Cherry SR, Sorenson JA, Phelps ME. Physics in Nuclear Medicine. 3rd ed. Philadelphia, PA: Saunders; 2003.

\section{Publish your work in this journal}

The International Journal of Nanomedicine is an international, peerreviewed journal focusing on the application of nanotechnology in diagnostics, therapeutics, and drug delivery systems throughou the biomedical field. This journal is indexed on PubMed Central, MedLine, CAS, SciSearch ${ }^{\circledR}$, Current Contents ${ }^{\circledR} /$ Clinical Medicine,
Journal Citation Reports/Science Edition, EMBase, Scopus and the Elsevier Bibliographic databases. The manuscript management system is completely online and includes a very quick and fair peer-review system, which is all easy to use. Visit http://www.dovepress.com/ testimonials.php to read real quotes from published authors. 